\title{
FEATURED ARTICLES
}

New kids on the block

Neuroscience Gateway (February 2008) | doi:10.1038/ngw1806

Neurogenesis in the adult brain is important in certain types of learning and memory.

In social networks, newcomers can affect the group dynamic. In neural networks, new neurons are generated and incorporated in discrete regions of the adult brain. How do these neurons affect mature brain function? Zhang et al. report that the proliferation of neural stem cells in the adult brain is important in spatial learning and memory in a recent article in Nature.

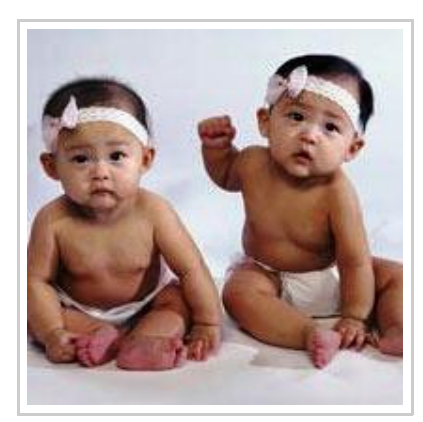

In the adult brain, neural stem cells generate new neurons in the subventricular zone and hippocampal dentate gyrus. Neural stem cells in the adult brain express the orphan receptor TLX. TLXpositive cells purified from the adult brain differentiate into neurons, astrocytes and oligodendrocytes, and neural stem cells with reduced $T / x$ expression show reduced proliferation in vitro. Traditional T/x knockout mice have unusually small brains (microencephaly) and are blind and aggressive.

To induce $T / x$ knockdown in adult mice, the authors generated mice with loxP sites flanking the $T / x$ gene $\left(T / x^{f / f}\right)$ and Cre recombinase expression under the control of a modified, tamoxifen-selective ligand-binding domain of the estrogen receptor $\left(C r e E R^{T M}\right)$. To control for hormone effects, the authors compared tamoxifen-treated $T / x^{f / f}$; CreER (Tlx-deficient) and $T / x^{f / f}$ (control) adult male mice. Bromodeoxyuridine (BrdU) labels DNA in dividing neurons. The authors treated adult mice with tamoxifen for 8 days, waited several weeks to several months and then treated them with BrdU for 6 days. Regardless of the time since treatment, Tlx-deficient mice showed fewer BrdU-labeled cells in the dentate gyrus relative to control mice, suggesting that TLX is important in proliferation of adult neural stem cells in vivo.

Does learning require neurogenesis? T/x-deficient and control mice froze with fear similarly in response to a shock-associated context or tone, suggesting that despite previous reports, blocking neurogenesis in the adult brain does not affect associative learning. However, Tlx-deficient mice showed deficits in spatial learning and memory. Mice do not like to swim. In the Morris water maze, mice learn the location of a platform submerged in opaque water. The authors used a challenging modification of the Morris water maze, in which the mice were not pretrained and had shorter swim trials. Tlx-deficient and control mice performed similarly on the first day of training. However, after several days of training, Tlx-deficient mice spent less time on or near the hidden platform than did control mice, suggesting that neurogenesis helps refine search strategies, according to the authors. Three weeks later, TIx-deficient mice performed similarly to control mice, suggesting that neurogenesis does not affect long-term memory. In reversal training, the platform is removed, and mice 'unlearn' its previous location. Relative to control mice, 
Tlx-deficient mice showed delayed reversal learning.

Therefore, neurogenesis affects the efficiency, not the effectiveness of spatial learning and regulates problem solving strategies, according to the authors. Although it is unclear what types of learning and memory are affected by neurogenesis in people, these data suggest that deficits in the production and incorporation of new neurons might be involved in certain learning disorders.

\section{Debra Speert}

1. Zhang, C.-L., Zou, Y., Gage, F. H. \& Evans, R. M. A role for adult TLX-positive neural stem cells in learning and

behaviour. Nature (2008)

doi: $10.1038 /$ nature06562. | Article | 